\title{
Sequential extraction of biogenic, authigenic and lithogenic silicas and silicates from marine sediments
}

\section{TZU HAO HUANG}

Stockholm University

Presenting Author: tzu-hao.huang@geo.su.se

Sequential extraction of biogenic, authigenic and lithogenic silicas and silicates from marine sediments

Tzu-Hao Huang, Wei-Li Hong, Xiaole Sun, Kalle Kirsimae, Peeter Somelar, Ji-Hoon Kim and Marta E. Torres

Silicon ( $\mathrm{Si}$ ) is the second most abundant element on Earth and plays a key role in the nutrient cycle and rock-forming process. In the global climate system, chemical weathering and reverse weathering of silicates significantly influences the carbonate system of the oceans and regulates the partial pressure of carbon dioxide in the atmosphere. Marine silicate weathering (MSiW) in anoxic sediment gained increased attention, with some reports suggesting that the carbon dioxide consumption rates through this process may be potentially as high as the rates of silicate weathering on land. However, the MSiW products and reactants have not yet been poorly quantified particularly with respect to the alteration products and their geochemical fingerprints in marine sediments. To move forward, it is important to develop analytical approaches that allow for the precise identification and quantification of individual Si-phases in marine sediments: biogenic, authigenic and lithogenic silica and silicates, including proper extraction, separation and characterization of these phases.

To this aim, here we described a sequential leaching protocol that can be used to separate the different Si-hosting phases in sediment samples collected from the Ulleung basin and Nankai subduction zone by scientific-ocean drilling expeditions. This protocol includes various extraction steps (two types of base leaching and alkaline fusion) that stepwisely leach biogenic silica, clays (both authigenic and lithogenic) and reactive lithogenic silicates such as feldspar and mica. We report on the concentrations of dissolved silicon together with other major and trace elements from the used leachates to assess the quality of the leaching protocol and provide quantitative information of the various Si components. Residual materials from the leaching of intermediate steps are collected and analyzed to identify the mineral assemblage by X-ray diffraction (XRD) and scanning electron microscopy (SEM). By combing chemical and mineralogical analytical results, we can elucidate the down core variations of various Si-phases and provide new insights into the overall scope of the transformations at play during the MSiW process. 